\title{
Abordagem transxifóidea sem esternotomia para correção da comunicação interatrial
}

\author{
Miguel BARBERO-MARCIAL*, Marcelo B. JATENE* ${ }^{*}$, Carla TANAMATI*, Nana Miura IKARI*, \\ Edmar ATIK*, Munir EBAID*, Geraldo VERGINELLI*, Adib D. JATENE*
}

RBCCV $44205-322$

Barbero-Marcial M, Jatene M B, Tanamati C, Ikari N M, Atik E, Ebaid M, Verginelli G, Jatene A D - Abordagem transxifóidea sem esternotomia para correção da comunicaçāo interatrial. Rev Bras Cir Cardiovasc $1996 ; 11(4): 287-91$.

RESUMO: Objetivo: Avaliação de uma nova abordagem cirúrgica para fechamento da comunicaçāo interatrial.

Métodos: A abordagem da comunicação interatrial através da janela xifóidea foi realizada em 6 pacientes do sexo masculino e 1 do sexo feminino e a idade variou de 6 meses a 14 anos, com média de idades de 5,1 anos. Em todos, foi utilizada a circulação extracorpórea, através de canulação da artéria femoral e átrio direito e videotoracoscopia para auxiliar a visibilização da aorta e do canal arterial, assim como das estruturas correspondentes às cavidades esquerdas.

Resultados: Não houve complicações intra-operatórias ou pós-operatórias e, em todos os pacientes, foi possivel a extubação na sala de operaçōes.

Conclusão: A janela xifóidea, sem a abertura do esterno, permitiu a correção da comunicação interatrial com bons resultados, podendo constituir-se em uma nova abordagem, minimamente invasiva.

DESCRITORES: Defeitos do septo interatrial, cirurgia. Osso xifóide. Circulaçāo extracorpórea. Toracoscopia. Cirurgia minimamente invasiva.

\section{INTRODUÇĀO}

A correção cirúrgica da comunicação interatrial e anomalias similares deveriam ser idealmente realizadas com o menor trauma cirúrgico, extubação do paciente na sala de operações, mínima dor e sem desconforto respiratório no pós-operatório, com tempo de internação aproximado de três dias. Conseqüentemente, redução do custo hospitalar.

O fechamento da comunicação interatrial através do cateterismo cardíaco tem sido feito em vários centros; por um lado, existem limitações quanto ao tipo e tamanho da CIA e, por outro, limitações, como a idade dos pacientes devido à via de acesso para introdução dos cateteres. Apesar dos inúmeros tipos de dispositivos e do grande esforço realizado pelos hemodinamicistas, este método não tem conseguido corrigir todos os defeitos atriais de forma definitiva, em todas as idades e sem complicações imediatas e tardias.

O objetivo do presente trabalho é apresentar uma nova abordagem cirúrgica, minimamente invasiva, sem abertura do esterno, para a correção de defeitos do septo interatrial.

Trabalho realizado no Instituto do Coração do Hospital das Clínicas da Faculdade de Medicina da Universidade de São Paulo. São Paulo, SP, Brasil. Recebido para publicação em novembro de 1996.

* Do Instituto do Coração do Hospital das Clínicas da Faculdade de Medicina da Universidade de São Paulo.

Endereço para correspondência: Miguel Barbero-Marcial. Av. Dr. Enéas de Carvalho Aguiar, 44. Divisão Cirúrgica. CEP: 05403-000, São Paulo, SP, Brasil. 
Barbero-Marcial M, Jatene M B, Tanamati C, Ikari N M, Atik E, Ebaid M, Verginelli G, Jatene A D - Abordagem transxifóidea sem esternotomia para correção da comunicaçäo interatrial. Rev Bras Cir Cardiovasc 1996; 11 (4):287-91.

\section{CASUÍSTICA E MÉTODOS}

No período de julho a outubro de 1996, foram operadas 6 crianças do sexo masculino e 1 do sexo feminino, com idades entre 6 meses e 14 anos, com média de 5,1 anos (Tabela 1 ).

A abordagem através da "janela xifóidea" foi realizada na seguinte seqüência:

- Incisão cutânea longitudinal de aproximadamente $5 \mathrm{~cm}$, na altura do apêndice xifóide.

- Resseç̧ão do apêndice xifóide e abertura de 1 $\mathrm{cm}$ com bisturi elétrico da porção supraxifóidea.

- Posicionamento do afastador de esterno, especialmente desenvolvido pela Divisão de Bioengenharia do Instituto do Coração do Hospital das Clínicas da Faculdade de Medicina da Universidade de São Paulo, de modo a tracionar superiormente e ampliar a exposição da "janela xifóidea" em $2 \mathrm{~cm}$ em direção cranial e $2 \mathrm{~cm}$ em sentido ventral (Figura 1).

- Abertura longitudinal do pericárdio.

- Dissecção da artéria femoral comum esquerda, para introdução da cânula arterial, através de incisão na pele de $2 \mathrm{~cm}$ de comprimento.

- Sutura em bolsa com Prolene 5-0 na face anterior da veia cava inferior.

- Sutura em bolsa com Prolene 5-0 na face lateral direita do átrio direito.

- Reparo das veias cavas com fita cardíaca.

- Ressecção de um segmento de $1 \times 1 \mathrm{~cm}$ de adventícia da face anterior da aorta para posterior infusão de cardioplegia e retirada de ar das cavidades esquerdas com auxílio da videoendoscopia.

Após heparinização $(4 \mathrm{mg} / \mathrm{kg})$ a cânula na arterial femoral (dlp) é introduzida e, a seguir, a cânula angulada na veia cava inferior e na cava superior, cânula aramada reta (Biotecno) (Figura 2). O calibre das cânulas é variável, adequado ao peso corpóreo: lactentes de até $5 \mathrm{~kg}$, cânula de aorta de $10 \mathrm{~mm}$ e cavas de $3 \mathrm{~mm}$ e $4 \mathrm{~mm}$; crianças de $5 \mathrm{~kg}$ a $10 \mathrm{~kg}$, cânula de aorta de $12 \mathrm{~mm}$ e cavas de $4 \mathrm{~mm}$; de $11 \mathrm{~kg}$ a $15 \mathrm{~kg}$, cânula de aorta de $14 \mathrm{~mm}$ e cavas de $3 / 16$ polegadas; de $16 \mathrm{~kg}$ a $25 \mathrm{~kg}$, cânula de aorta de $16 \mathrm{~mm}$ e cavas de $3 / 16$ polegadas; de $25 \mathrm{~kg}$ a $40 \mathrm{~kg}$, cânula de aorta $18 \mathrm{~mm}$ e cavas de $1 / 4$ de polegada.

- Início da circulação extracorpórea normotérmica com pinçamento transversal da aorta passando - "braço" inferior da pinça pelo seio transverso.

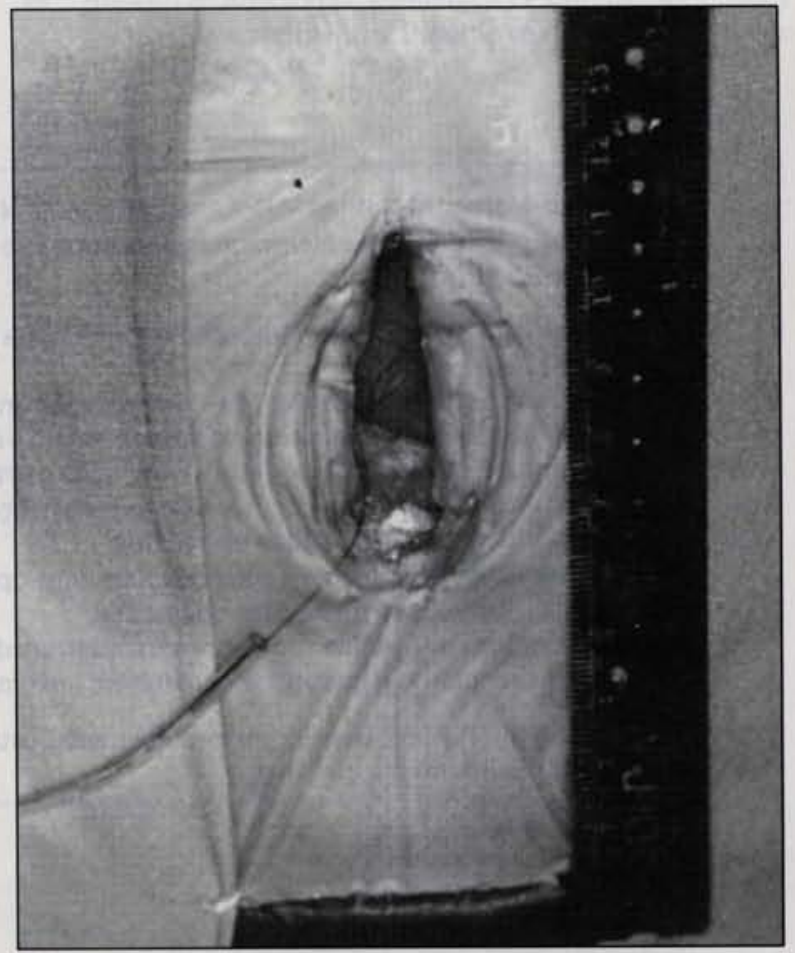

Fig. 1 - Fotografia mostrando a incisāo transxifóidea de $5 \mathrm{~cm}$ e visão parcial da face diafragmática do ventrículo direito. $O$ fio de Prolene 5-0 (Ethicon) reparado com um tubo de plástico foi utilizado para sutura em bolsa do átrio direito, para canulação da veia cava superior.

TABELA 1

DADOS CLINIICOS

\begin{tabular}{crrrrrrrr}
\hline CASO & IDADE & $\begin{array}{c}\text { TIPO } \\
\text { DEEFEITO }\end{array}$ & INCISÃO & TCEC & Tpinçam & EXTUBAÇÃO & ALTA & COMPLICAÇŌES \\
\hline 1 & 5 anos & CIAOS & $10 \mathrm{~cm}$ & $28 \mathrm{~min}$ & $14 \mathrm{~min}$ & SALA & PO $5 \mathrm{~d}$ & Nenhuma \\
2 & 14 anos & CIA OS & $12 \mathrm{~cm}$ & $19 \mathrm{~min}$ & $11 \mathrm{~min}$ & SALA & PO $4 \mathrm{~d}$ & Nenhuma \\
3 & 5 anos & CIA OS & $7 \mathrm{~cm}$ & $16 \mathrm{~min}$ & $5 \mathrm{~min}$ & SALA & PO $5 \mathrm{~d}$ & Nenhuma \\
4 & 4 anos & CIAOS & $6 \mathrm{~cm}$ & $21 \mathrm{~min}$ & $12 \mathrm{~min}$ & SALA & PO $5 \mathrm{~d}$ & Nenhuma \\
5 & 6 meses & CIA OS & $5 \mathrm{~cm}$ & $17 \mathrm{~min}$ & $9 \mathrm{~min}$ & SALA & PO $4 \mathrm{~d}$ & Nenhuma \\
6 & 8 meses & CIA OS & $5 \mathrm{~cm}$ & $29 \mathrm{~min}$ & $20 \mathrm{~min}$ & SALA & PO $3 \mathrm{~d}$ & Nenhuma \\
7 & 7 anos & CIAOS & $7 \mathrm{~cm}$ & $31 \mathrm{~min}$ & $17 \mathrm{~min}$ & SALA & PO $4 \mathrm{~d}$ & Nenhuma \\
\hline
\end{tabular}

$\mathrm{CIA}$ : comunicação interatrial; OS: ostium secundum; m: meses; d: dias; T pinçam.: tempo de pinçamento aórtico; T CEC: tempo de circulação extracorpórea. 
Barbero-Marcial M, Jatene M B, Tanamati C, Ikari N M, Atik E, Ebaid M, Verginelli G, Jatene A D - Abordagem transxifóidea sem esternotomia para correção da comunicação interatrial.

- O átrio direito é aberto, paralelo ao sulco atrioventricular e as bordas reparadas e tracionadas.

- O fechamento da comunicação interatrial é realizado da maneira convencional, sem esvaziar o átrio esquerdo.

- Com auxílio da cardioscopia, um ponto em "U" de Prolene 5-0 é dado na parede da aorta previamente liberada da adventícia, proximal à pinça da aorta. A aorta é puncionada com agulha hipodérmica $(30 \mathrm{~mm} \times 8 \mathrm{~mm}$ ) com reparo de plástico ou de borracha colocado a $1 \mathrm{~cm}$ da ponta, para retirada de ar e sangue das cavidades esquerdas.

- A pinça da aorta é retirada e o átrio direito fechado com dupla sutura de Prolene 5-0.

- Após a saída de circulação extracorpórea, as cânulas são retiradas e o mediastino é drenado.

- O pericárdio é fechado parcialmente com pontos separados de algodão $2-0$, e os planos da parede são fechados de modo convencional.

O tempo de circulação extracorpórea variou de $16 \mathrm{~min}$ a $31 \mathrm{~min}$ (média de $21,57 \mathrm{~min}$ ) e de pinçamento aórtico de $5 \mathrm{~min}$ a $20 \mathrm{~min}$ (média de $12,57 \mathrm{~min})$.

\section{RESULTADOS}

Nos 7 pacientes operados, a abordagem xifóidea permitiu o acesso à correção cirúrgica. Todos os pacientes foram extubados em sala cirúrgica e evoluíram sem intercorrências no pós-operatório, recebendo alta hospitalar entre $02^{\circ}$ dia e $05^{\circ}$ dia. O ecocardiograma transtorácice realizado no pósoperatório mostrou correção total dos defeitos. A canulação arterial não alterou o fluxo ou o pulso do membro inferior correspondente.

\section{COMENTÁRIOS}

O interesse pelo desenvolvimento de técnicas cirúrgicas menos invasivas, através de incisões cada vez menores e com o auxílio de equipamentos de videoendoscopia vem aumentando progressivamente. O maior conforto para o paciente, o menor tempo de internação hospitalar e o menor número de complicações pós-operatórias justificam a tendência atual. Na cirurgia cardiaca, algumas operaçōes, denominadas minimamente invasivas, têm sido realizadas para revascularização do miocárdio sem circulação extracorpórea (1) e em trocas valvares aórticas e mitrais ${ }^{(2)}$ com auxílio da videoendoscopia, geralmente utilizadas para biópsia e drenagem pericárdica, fechamento de canal arterial e secção e sutura de anel vascular pulmonar $(3,4)$.

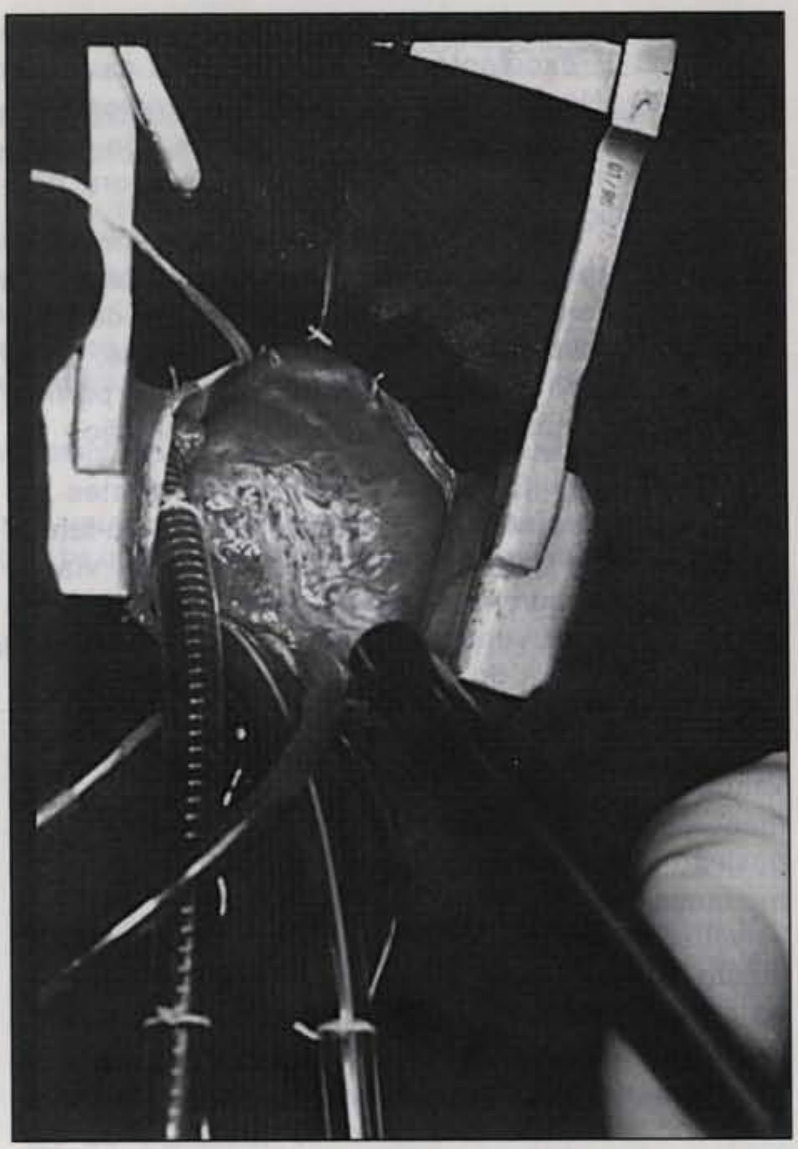

Fig. 2 - Campo cirúrgico da abordagem transxif́́idea com o afastador de esterno desenvolvido pela Bioengenharia do Incor $e$ as cânulas de extracorpórea posicionadas - cava superior (cânula aramada) e inferior. O toracoscópio, situado inferiormente e à direita, é utilizado para visibilização das câmaras cardiacas esquerdas e aorta.

$\mathrm{Na}$ cirurgia cardiaca pediátrica, existem dificuldades para realização das operações menos invasivas devido à complexidade das cardiopatias, baixo peso corpóreo e a manipulação de estruturas anatômicas extremamente delicadas. CHANG et al. (5) publicaram, recentemente, os resultados do fechamento da CIA com videotoracoscopia, através de minitoracotomia ântero-lateral direita; o defeito foi corrigido, porém com hipotermia profunda e longos períodos de circulação extracorpórea (47 min a $126 \mathrm{~min}$ ). Apesar da boa evolução, a técnica transforma uma operação simples e segura numa outra com maiores dificuldades, maior tempo de circulação extracorpórea e riscos potenciais de embolia aérea.

Através da janela xifóidea, pôde-se realizar com segurança a correção da comunicação interatrial. As vantagens em não abrir o esterno estão no período de pós-operatório, com a rápida recuperação, menos dor e menor desconforto. A alta hospitalar pode ser efetuada no $3^{\circ}$ dia de pós-operatório, 
Barbero-Marcial M, Jatene M B, Tanamati C, Ikari N M, Atik E, Ebaid M, Verginelli G. Jatene A D - Abordagem transxifóidea sem esternotomia para correção da comunicação interatrial,

Rev Bras Cir Cardiovasc 1996; 11 (4):287-91

com reintegração às atividades físicas normais quase imediata. $O$ excelente aspecto estético deve ser ressaltado. Nos primeiros 6 casos, preferimos manter o paciente internado até o $5^{\circ}$ dia de pós-operatório, para melhor acompanhamento, embora houvesse condições de alta no $3^{\circ}$ dia.

A videotoracoscopia não é indispensável para a operação. No entanto, permite, em casos de dúvidas, uma visão perfeita dos vasos da base, veias cavas e o ducto arterioso, sendo a forma de permitir que os assistentes acompanhem a operação.

Durante muito tempo a abordagem destes defeitos foi feita através de toracotomia ântero-lateral na altura do $4^{\circ}$ espaço intercostal (6). Esta via de acesso, adequada para corrigir os defeitos do septo interatrial e interventricular alto, tem os inconvenientes da abertura do espaço pleural, maior incidência de atalectasias e outras complicações pulmonares, e maior dor no pós-operatório por trauma dos vasos intercostais. Por outro lado, em crianças menores de um ano de idade é difícil determinar a posição exata do sulco submamário, já que existem mudanças na sua posição de acordo com o desenvolvimento torácico e, em mulheres, o desenvolvimento das mamas. Em alguns casos de lactentes, na nossa experiência, a desarticulação ou o rompimento da cartilagem intercostal provocada pelo afastador de tórax tem levado tardiamente a diferença no crescimento dos arcos costais e da cartilagem previamente lesada, com deformidade torácica permanente, embora mais rara em crianças maiores e adultos.

A incisão cutânea submamária bilateral $(7,8)$ para realizar a esternotomia é de extrema importância do ponto de vista estético e tem sido usada durante anos, em nosso Serviço, em pacientes do sexo feminino; demanda um extenso descolamento do tecido celular subcutâneo e das glândulas mamárias, embora com pequeno número de complicações. As complicações da esternotomia são bem conhecidas. Apesar da correta aproximação das bordas do esterno com fios de aço, a esternotomia mediana provoca instabilidade torácica com repercussões na mecânica ventilatória e dor torácica, que se acentua com os movimentos e a tosse.

Outras vias de acesso foram desenvolvidas para a abordagem minimamente invasiva de defeitos intracardíacos, como a esternotomia reduzida, com abertura parcial do esterno, como descrita por TATEBE et al. (9), WILSON Jr. et al (10) e KOMAl et al. (11).

Nos últimos anos, várias tentativas têm sido realizadas para fechar por cateterismo as comunicações interatriais, interventriculares e a persistência de canal arterial através de diversos tipos de dispositivos, como a mola de Gianturco, clamp-shell ou guarda-chuvas (12-15). As complicações imedia-

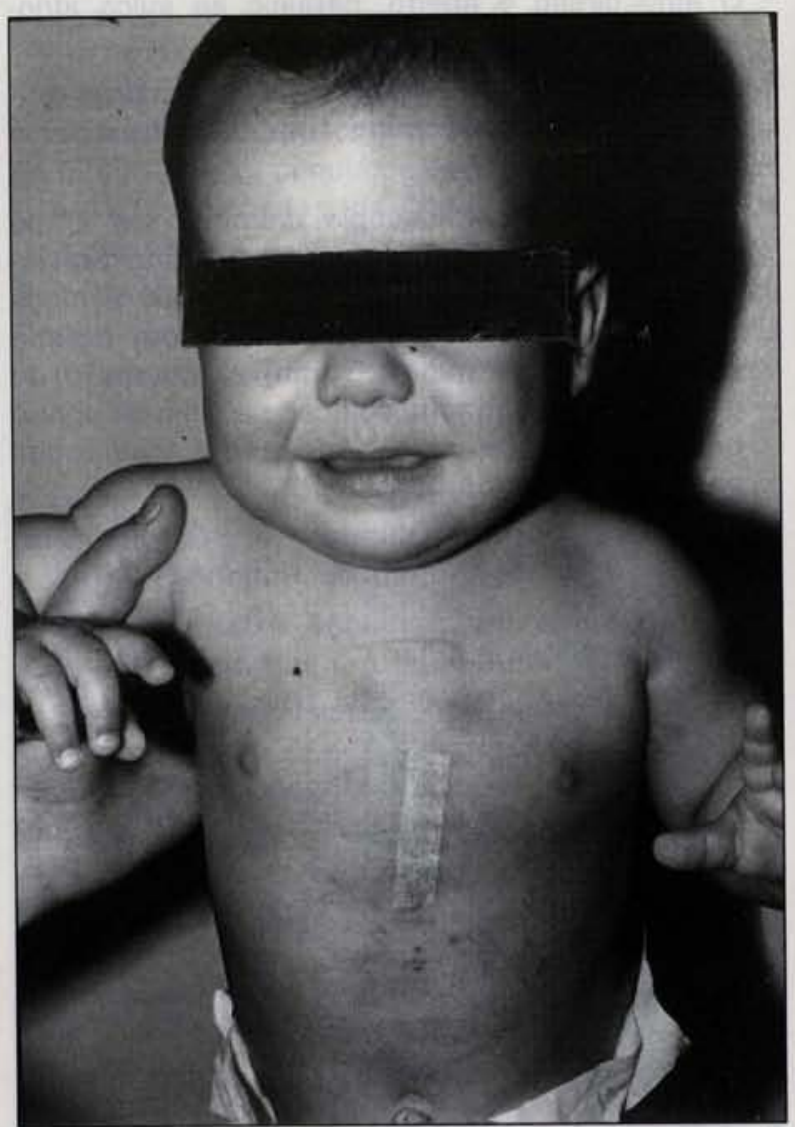

Fig. 3 - Lactente de 8 meses, no $7^{\circledR}$ dia de pós-operatório, mostrando o tamanho da incisāo transxifóidea delimitada pelo curativo com micropore.

tas e tardias levaram à suspensão dos procedimentos para fechamento da CIA por cateterismo, pois as hastes do dispositivo utilizado sofriam fraturas podendo causar embolias pulmonares. Novos tipos de dispositivos têm sido criados e testados experimentalmente e, no futuro, poderemos avaliar sua eficácia. Outros fatores limitantes são o tamanho e - tipo da comunicação interatrial, e a dificuldade para acesso venoso ou arterial em crianças de baixo peso e suas complicações, como a trombose venosa profunda e a isquemia arterial do membro.

A abordagem utilizada, com as vantagens já discutidas, torna-se opção válida para a correção de defeitos intracardíacos simples como fechamento das comunicações interatriais (Figura 3). A evolução hospitalar favorável, o menor custo hospitalar e a estética da incisão motivam o seu emprego em pacientes portadores destas anomalias. No entanto, deve ser mencionado o aumento da dificuldade técnica diante da pequena incisão utilizada; serão necessários treinamento cirúrgico e cuidados na manipulação do coração; na presença de qualquer complicação, não hesitar em realizar a operação através da esternotomia mediana. 
Barbero-Marcial M, Jatene M B, Tanamati C, Ikari N M, Atik E, Ebaid M, Verginelli G, Jatene A D - Abordagem transxifóidea sem esternotomia para correçăo da comunicação interatrial. Rev Bras Cir Cardiovasc 1996; 11 (4):287-91.

\section{RBCCV 44205-322}

Barbero-Marcial M, Jatene MB, Tanamati C, Ikari N M, Atik E, Ebaid M, Verginelli G, Jatene A D - Transxiphoid approach without sternotomy for the correction of atrial septal defect. Rev Bras Cir Cardiovasc 1996; $11(4): 287-91$.

ABSTRACT: Purpose: Evaluate a new access for surgical correction of atrial septal defects.

Methods: The access by xiphoid window was done in 7 cases with atrial septal defect type ostium secundum. The age ranged from 6 months to 14 years, mean-age 5.1 years old. In all cases the extracorporeal circulation was done with femoral artery and right atrial cannulation and aortic clamping. The video-assisted endoscopy was utilized to help the visualization of the aorta, ductus arteriosus and the anatomy of the left intracardiac structures.

Results: There was no intraoperative or postoperative complications and in our 7 cases the extubation at the operating room was possible.

Conclusion: The xiphoid window without opening the sternum permited correction of the atrial septal defect with good results and without complications, and could be used as a new technique, less invasive, to correct these defects.

DESCRIPTORS: Atrial heart septal defects, surgery. Xiphoid bone. Extracorporeal circulation. Thoracoscopy. Surgery, minimally invasive.

\section{REFERÊNCIAS BIBLIOGRÁFICAS}

1 Benetti F J \& Ballester C - Use of thoracoscopy and a minimal thoracotomy, in mammary-coronary bypass to left anterior descending artery, without extracorporeal circulation: experience in 2 cases. $J$ Cardiovasc Surg 1995; 36: 159-61.

2 Lin $\mathrm{P} \mathrm{J}$, Chang $\mathrm{C} \mathrm{H}$, Chu $\mathrm{J} \mathrm{J}$ et al. - Video-assisted mitral valve operations. Ann Thorac Surg 1996;61: $1781-7$.

3 Burke R P, Wernovsky G, van der Velde M, Hansen D, Castañeda A R - Video-assisted thoracoscopy surgery for congenital heart disease. $J$ Thorac Cardiovasc Surg 1995; 109: 499-508.

4 Burke R P \& Chang A C - Video-assisted thoracoscopic division of a vascular ring in an infant: a new operative technique. J Thorac Cardiovasc Surg 1993; 8: 537 40.

5 Chang $\mathrm{C} \mathrm{H}$, Lin $\mathrm{P} J$, Chu $\mathrm{J} \mathrm{J}$ et al. - Video-assisted cardiac surgery in closure of atrial septal defect. Ann Thorac Surg 1996; 62: 697-701.

6 Rosengart T K \& Stark J F - Repair of atrial septal defect through a right thoracotomy. Ann Thorac Surg 1993; 55: $1138-40$.

7 Laks $\mathrm{H}$ \& Hammond $\mathrm{G}$ - A cosmetically acceptable incision for the medial sternotomy. J Thorac Cardiovasc Surg 1980; 79: 146-9.
8 Baucia J A, Ferreira Sobrinho A, Barbero-Marcial M. Incisāo submamária para esternotomia mediana. Arq Bras Cardiol 1993; 60: 335-8.

9 Tatebe S, Eguchi S, Miyamura $\mathrm{H}$ et al. - Limited vertical skin incision for median sternotomy. Ann Thorac Surg 1992; 54: 787-8.

10 Wilson Jr. W R, Ibawi M N, DeLeon S Y et al. - Partial median sternotomy for repair of heart defects: a cosmetic approach. Ann Thorac Surg 1992; 54: 892-93.

11 Komai $\mathrm{H}$, Naito $\mathrm{Y}$, Fujiwara K et al. - Lower mid-line skin incision and minimal sternotomy: a more cosmetic challenge for pediatric cardiac surgery. Cardiol Young 1996; 6: 76-9.

12 King T D \& Mills N L - Secundum atrial septal defect: nonoperative closure during cardiac catheterization. JAMA 1976; 235: 2506.

13 Latson L A, Hofschire P J, Kugler J D et al. - Residual shunts after transcatheter closure of patent ductus arteriosus: a major concern of benign "technomalady"?. Circulation 1991; 84: 2591-3.

14 Rome J J, Keane J F, Perry S B, Spevak P J, Lock J E - Double-umbrella closure of atrial septal defects: initial clinical applications. Circulation 1990, 82: 751 8.

15 Rao P S, Wilson A D, Chopra P S - Transcatheter closure of atrial septal defect by "buttoned" devices. Am J Cardiol 1992; 69: 1056-61. 